\title{
The PRISMA-LHAASO project: Status and overview
}

\author{
Victor Alekseenko ${ }^{1}$, Anastasia Bagrova ${ }^{1,2}$, Shuwang Cui ${ }^{3}$, a , Yayun $\mathrm{He}^{3}$, Bingbing $\mathrm{Li}^{3}, \mathrm{Xinhua} \mathrm{Ma}^{4}, \mathrm{Egor} \mathrm{Pozdnyakov}^{5}$, \\ Oleg Shchegolev ${ }^{1}$, Yuri Stenkin ${ }^{1,2}$, and Vladimir Stepanov ${ }^{1}$ \\ 1 Institute for Nuclear Research, Russian Academy of Sciences, Russia \\ 2 Nuclear Research National University "Moscow Engineering Physics Institute", Russia \\ 3 The College of Physics and Information Engineering, Hebei Normal University, China \\ ${ }^{4}$ Key Laboratory of Particle Astrophysics, Institute of High Energy Physics, Chinese Academy of Sciences, China \\ 5 CJSC NPF "Luminofor", Russia
}

Abstract. The status of and main features of the PRISMA-LHAASO project are presented. We discuss here the science project and some preliminary results obtained with the PRISMA prototype.

\section{Introduction: The PRISMA-LHAASO project science}

There exist (or existed) very few experiments specially designed to solve the 60 years old "knee problem" in cosmic ray. The best of them, namely, KASCADE and Tibet AS gave very precise and interesting but contradictory results and did not solve the problem [1]. It became even less clear. Neither the knee origin nor the cosmic ray energy spectrum and its mass composition are known today. These problems could not be solved in our opinion by a traditional Extensive Air Shower (EAS) method studying electromagnetic and muon components. New approaches [2] based on the new ideas and multicomponent EAS analysis will help us to solve this complicated problem.

The idea of a novel type of array for EAS study in 2001 [3] has been developed in 2008 to the PRISMA (PRImary Spectrum Measurement Array) project [4,5]. It is based on a simple idea: hadrons are the main EAS component forming its skeleton and resulting in all its properties at an observational level [6], so the hadron component should also be the main component to be measured in experiments. During its longitudinal developing in the atmosphere the EAS passes through several stages from the first cosmic ray interaction to almost full degradation deep in the atmosphere. Only the hadron content can be used to recognize the stage of EAS development. The development of a novel type of EAS array detector (en-detector) [7], able to record the hadronic component through thermal neutron detection and electronic component as well, can help to solve the problem.

The experiment has to be performed at high altitude where the number of secondary hadrons reaches maximal. The detector looks like an ordinary EAS detector but with a specific thin inorganic scintillator sensitive to thermal neutrons and having low sensitivity to charged

\footnotetext{
a e-mail: cui_shuwang@163.com
}

particles. A thin layer of scintillator consists of an alloy of natural boron salt with inorganic scintillator $\mathrm{ZnS}(\mathrm{Ag})$. The recording efficiency of the compound to thermal neutrons is close to $20 \%$. By spreading these detectors over a large area on the Earth's surface one can obtain a kind of hadronic calorimeter of practically unlimited area. To make the energy threshold of the array lower it has to be installed at high altitude. We proposed to locate the PRISMA array at $4400 \mathrm{~m}$ above sea level in the central part of the LHAASO array.

The PRISMA-LHAASO project is mostly aimed to solve the "knee problem" in the cosmic ray spectrum and to measure any changes of cosmic ray mass composition at energies in the $\mathrm{PeV}$ region. The best way to do so could be direct cosmic ray spectrum measurements. Unfortunately, it cannot be performed due to the very low intensity of cosmic rays with energies above $1 \mathrm{PeV}$. That is why physicists are pressed to use an indirect EAS method. However, as a payment for that, one has to make very complicated and model dependent recalculations from the measurements to primary parameters. Solving the inverse task one should be sure that: i) a solution exists and ii) the measured parameters are connected with real ones by known dependencies. Both points are not known $a$ priori. Solving the direct task one also has to introduce many parameters by hand, concerning the details of the model used, cosmic ray mass composition, existence or presence of the "knee" in the primary spectrum etc. [8].

Traditionally EAS arrays measure the electron component first of all. This is not the best but the simplest and most convenient choice because the electron component is the most numerous. However, the electron component is the secondary EAS component sensitive to EAS longitudinal development, which is formed by the cascading of high-energy hadrons. These two components are in a dynamic equilibrium. However, the equilibrium exists only while hadrons exist. When the cascading hadrons are fully exhausted (note that the number of such hadrons is high only at the maximum of shower development and then exponentially decreases with depth 


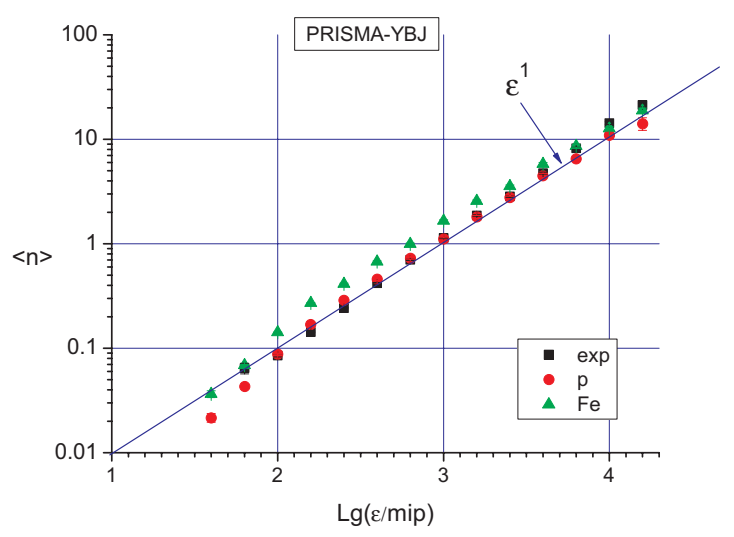

Figure 1. Correlation plot between electromagnetic EAS component (total array energy deposit $\epsilon$ ) and the mean number of recorded thermal neutrons $\langle\mathrm{n}\rangle$.

in the atmosphere), the equilibrium violation occurs. This occurs at energy $100 \mathrm{TeV} /$ nucleon. It changes the EAS properties dramatically and results in a visible break in the electromagnetic components (including Cherenkov light) $[2,8]$. Interpretation of the data obtained with traditional EAS arrays is very complicated and ambiguous. Therefore, the best way is to record the primary EAS component, namely the hadronic one, along with other components. The PRISMA experiment will realize this approach measuring hadrons (thermal neutrons) over the whole array area $100 \times 100 \mathrm{~m}^{2}$. In fact, it can be regarded as a kind of huge area single layer hadronic calorimeter, which can be extended without any problem.

\section{The PRISMA-LHAASO array details}

The array will consists of 25 independent clusters, 16 en-detectors each. The cluster geometry is very simple: a homogeneous rectangular grid with $5 \mathrm{~m}$ spacing covering $15 \times 15 \mathrm{~m}^{2}$. Each cluster will work independently triggering by a 2 -fold coincidence of any 2 hit detectors with a threshold of 5 particles (m.i.p.). All clusters will be synchronized with each other and so the full array data combination and processing will be made off-line. We will use two signals from each detector: from the last and the 5th dynodes to extend the pulse height measurement range up to $\sim 10000$ particles. The dynamic range for neutron measurements is close to 1000 in each detector (or 16000 in total) inside a time gate of $20 \mathrm{~ms}$ after EAS passage. All pulses will be digitized using 32-channels and 16-bits FADC. For all powerful showers the full $20 \mathrm{~ms}$ waveforms are stored on disc with $1 \mu$ s time resolution. This allows us to check and analyze the selected events visually.

The daily energy deposit spectra of each detector are stored to hard disc to control its gain and stability. Moreover, the time series of the counting rate for events selected by pulse shape analysis as "neutrons" and those as "charged", are stored every $5 \mathrm{~min}$. These data are used for the environmental neutron flux variations study. The latter also allows us to carry out geophysical research, such as neutron and radon diurnal and seasonal waves, thunderstorms, tidal waves, seismic activity, free Earth's oscillations, etc. (see below).

\section{Some results of EAS study with a prototype}

The PRISMA prototype array made with 4 en-detectors (PRISMA-YBJ) has been running in Tibet (Yangbajing, $4300 \mathrm{~m}$ a.s.1.) for more than 3 years and some interesting results have been obtained with it up to date. One can see the correlation plot between EAS electromagnetic and neutron components in Fig. 1 in comparison with the experiment Monte-Carlo simulations for primary protons and iron nuclei. No normalization is applied and the agreement is good. Unfortunately, this plot depends on the absolute values and is weakly sensitive to a mass composition and therefore, it can not be successfully used for such measurements. We used CORSIKA code (ver. 6900, QGSJET, Gheisha) for EAS simulation and GEANT4.10 for the en-detector response simulations [9, 10]. It is seen that at least at high altitude close to EAS maximum, the dependence can be well fitted with a linear function.

Many other results obtained with our prototypes can be found elsewhere [11,12].

\section{Geophysical researches with environmental neutrons flux}

Environmental neutrons are produced by two natural sources: by cosmic rays in air and in the upper layers of soil and by natural radioactivity (mostly due to $(\alpha, \mathrm{n})$-reactions on light nuclei) throughout the Earth's crust. Fast produced neutrons are then moderated by media up to thermal energy and live there up to nuclear capture. Neutron lifetime depends on the media's chemical composition, temperature and water (or any hydrogenous material) content. The natural radioactive chain daughter product inert gas radon-222, having $3.8 \mathrm{~d}$ half-life, can migrate long distances in air and in soil (rock, concrete, etc.) and even accumulate in some places thus changing the neutron flux in underground locations. It is also sensitive to local seismic activity. Therefore, the flux (or concentration) of thermal neutrons in the media is sensitive to the media parameters such as its temperature, humidity, porosity (seismic activity), etc. Therefore, measuring neutron flux variations for a long time could be used to control the above media parameters.

We plan to use the en-detectors of PRISMA-LHAASO array for continuous environmental thermal neutron flux monitoring and its variations, a study needed not only for EAS experiment background estimation but also for some geophysical applications. The following geophysical items will be investigated through thermal neutron studies with the en-detectors installed underground or on the surface:

- neutrons during thunderstorms (surface)

- Earth's crust Moon tidal effects (surface and underground)

- Seasonal radon-neutron waves at high altitude (surface)

- Free Earth's oscillations (underground)

- Forbush effect and Sun-Earth interconnections (surface and underground)

- Ground Level Enhancement (GLE) effect (surface).

The additional geophysical studies on the Earth's surface could be performed cost free using PRISMA-LHAASO 


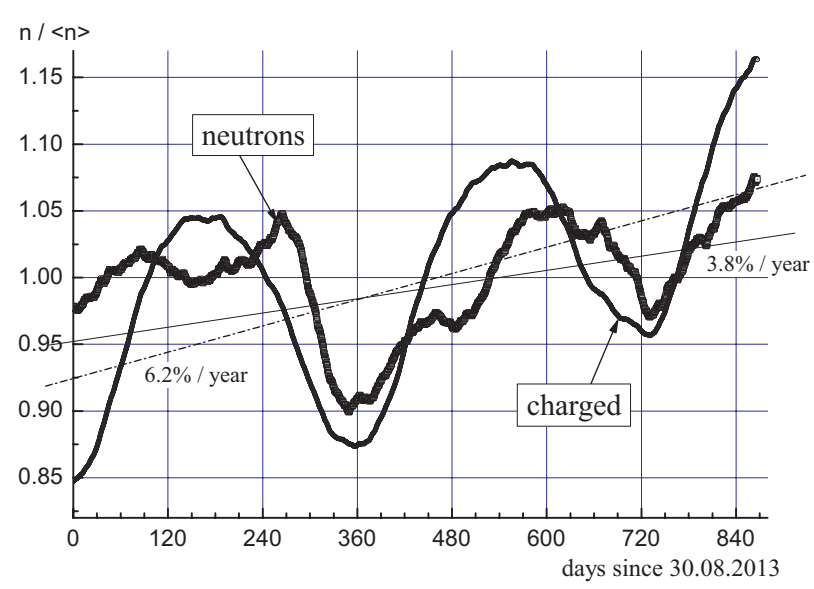

Figure 2. Long-term smoothed variations of thermal neutron background at Yangbajing.

detectors. The items need an underground detector location and could use existing underground or basement rooms to decrease the cosmic ray background and to emphasize the radon-neutron source. Otherwise, it will need additional investments.

An example of thermal neutron variations study can be seen in Fig. 2 where a normalized and 60-day smoothed counting rate is shown for both neutrons and for "charged" for about a 3y-period of observation at Yangbajing. Seasonal variations of about $8 \%$ amplitude wave anticorrelated with the rainy season is clearly visible in both curves [13]. These data allows us to estimate the neutron background at high altitude. As we have found the neutron background has also a long-term trend, which we connected with the decrease of solar activity in the 24th solar cycle, resulting in an increase of low energy cosmic rays producing neutrons [14].

More results concerning geophysical studies can be found elsewhere $[13,15,16]$.

\section{Summary}

A novel type of EAS full-scale array (PRISMA-LHAASO) will be made at high altitude in association with the LHAASO project in the next 4 years. Preliminary results obtained with the prototypes and $\mathrm{M}-\mathrm{C}$ simulations make us sure that en-detectors are very informative and very stable and the future array could give us absolutely new and fascinating results on cosmic rays near and above so-called "knee" in cosmic ray spectrum. We hope that the problem of the "knee" will be solved soon with the PRISMA-LHAASO.

This work was supported in Russia by RFBR (grants 14-0200996, 16-32-00054 and 16-29-13067_ofi_m), RAS Presidium Program "Fundamental properties of matter and astrophysics", and in China by NSFC (No. 10975046, No. 11375052).

\section{References}

[1] M. Amenomori et al., Astroph. J. 678, 1165 (2008)

[2] Yu.V.Stenkin, Mod. Phys. Lett. A 8, 18, 1225-1234 (2003)

[3] Yu.V. Stenkin and J.F. Valdes-Galicia, Proc. of 27th ICRC, 1453 (2001)

[4] Yu.V. Stenkin, Nucl. Phys. B (Proc. Suppl.) 175-176, $326(2008)$

[5] Yu.V. Stenkin, Nucl. Phys. B (Proc. Suppl.) 196, 293-296 (2009)

[6] G.T. Zatsepin, Docl. AN SSSR 67, 993 (1949)

[7] Yuri V. Sten'kin. LARGE SCINTILLATOR DETECTOR FOR THERMAL NEUTRON RECORDING. In: Nuclear Track Detectors: Design, Methods and Applications ISBN: 978-1-60876-826-4, Editor: Maksim Sidorov and Oleg Ivanov (c) 2010 Nova Science Publishers, Inc. 10, 253-256.

[8] Yu. V. Stenkin, Physics Atomic Nucl. 71, 1, 98 (2008)

[9] Y.V. Stenkin et al., Chinese Physics C 37, 1, 015001 (2013)

[10] O.B. Shchegolev and Yu.V. Stenkin, Bulletin of the Lebedev Physics Institute 43, 7, 223 (2016)

[11] B. Bartoli et al., Astroparticle Physics 81, 49-60 (2016)

[12] O. B. Shchegolev et al., J. Phys.: Conf. Ser. 718, 052038 (2016)

[13] Yu.V. Stenkin et al., Pure and Appl. Geophys., in press; arXiv: 1605.01283 [physics.geo-ph]

[14] Yu.V. Stenkin et al., Bulletin of Russian Academy of Sciences, in press (2017)

[15] V. Alekseenko et al., Phys. Rev. Lett. 114, 125003 (2015)

[16] V. Alekseenko et al., J. Phys.: Conf. Ser. 409, 012190 (2013) 